Anita Santner*

\title{
Das SLUB TextLab: Offene Werkstatt für analoge und digitale Textarbeit
}

https://doi.org/10.1515/bfp-2020-2092

Zusammenfassung: Auf der Suche nach einem neuen Raumnutzungskonzept für die Zweigbibliothek Erziehungswissenschaft wurde an der SLUB Dresden in den letzten zwei Jahren das SLUB TextLab entwickelt. In $\mathrm{Zu}-$ sammenarbeit mit lokalen Kooperationspartnern unterstützt es Nutzer bei der analogen und digitalen Textarbeit. Die Kombination von Schreibberatung, Digital Humanities und Embodiment in einer wissenschaftlichen Bibliothek als Dritter Ort ist innovativ und zukunftsrelevant, da Bibliotheken aufgrund der zunehmenden Digitalisierung und dem damit verbundenen Rückgang des physischen Bestandes ein neues Profil entwickeln müssen.

Schlüsselwörter: Textwerkstatt; TextLab; Digital Humanities; Embodiment; Schreibberatung; Dresden

\section{Introducing SLUB TextLab: Open Workshop for Analogue and Digital Textual Work}

Abstract: In search for an adapted room utilization concept for the branch library of education at the SLUB Dresden the SLUB TextLab has been developed over the past two years. In collaboration with local partner institutions it offers library users support with analogue and digital text production and analysis. The combination of writing counselling, digital humanities, and embodiment in a research library conceived of as a third place is innovative and relevant for the future, as libraries have to develop a new profile in an increasingly digitalized world.

Keywords: TextLab; digital humanities; embodiment; writing counselling; textual makerspace; Dresden

\section{Zur Entstehungsgeschichte des Projekts}

Schreibberatung hat sich in den letzten Jahren zu einem wichtigen Baustein im Serviceportfolio wissenschaftlicher Bibliotheken und hochschuldidaktischer Zentren an Universitäten im deutschsprachigen Raum entwickelt. An

*Kontaktperson: Anita Santner, anita.santner@slub-dresden.de der Sächsischen Landesbibliothek, Staats- und Universitätsbibliothek (SLUB) Dresden ist die individuelle Nutzerberatung zu Themen rund um den Schreib- und Publikationsprozess seit Jahren in der SLUB Wissensbar ${ }^{1}$ institutionalisiert und fixer Bestandteil des Serviceangebots. Als die TU Dresden im Rahmen ihrer Bewerbung zur Exzellenzuniversität 2016 auf diesem Gebiet nachzog und gleich zwei Einrichtungen gründete, die universitäre Schreibberatung anbieten - das Schreibzentrum (SZD) und die Schreibwerkstatt der Graduiertenakademie - initiierte die SLUB das Kooperationsmodell Netzwerk Schreiben, um diese Angebote zu bündeln ${ }^{2}$ und die genannten Einrichtungen unter einem gemeinsamen Dach - zunächst virtuell - zu versammeln. Durch die „Ausdifferenzierung des Portfolios“ gelang es den beteiligten Institutionen, „Konkurrenz $\mathrm{zu}$ vermeiden und stattdessen kooperativ Synergien zu nutzen“. ${ }^{3}$ Das Ergebnis dieses Prozesses war ein gemeinsamer Veranstaltungskalender auf der Webseite der SLUB, in dem seit 2017 sämtliche Angebote zur Schreibberatung der involvierten Akteure zentral erfasst und mit einer einheitlichen Anmeldefunktion versehen sind. ${ }^{4}$

Während das Netzwerk Schreiben im dem gemeinsamen Veranstaltungskalender also rasch eine digitale Heimat gefunden hatte, war eine analoge Entsprechung in Form eines gemeinsamen Schreibraums noch nicht gegeben. Um die akademische Schreibberatung verstärkt in das Curriculum zu integrieren, fanden seit dem Sommersemester 2017 in Zusammenarbeit mit der TU Dresden Lehrveranstaltungen zur Vermittlung von Schreibkompetenz statt. In Ermangelung eines gemeinsamen Schreibraums wurde dafür auf die Schulungsräume der SLUB und auf den in der Zweigbibliothek DrePunkt angesiedelten Makerspace zurückgegriffen. ${ }^{5}$ Zwischen diesen beiden Varianten bevorzugten die Teilnehmenden die Verortung der Schreibwerkstatt im Makerspace gegenüber den Schulungsräumen. Meyer kommt $2018 \mathrm{zu}$ folgendem Schluss:

\footnotetext{
1 Vgl. Mittelbach (2013).

2 Vgl. Meyer (2018) 289.

3 Meyer (2018) 294.

4 Meyer (2018) 295.

5 Vgl. Meyer (2018) $295 \mathrm{ff}$.
} 
„Trotz der regen Nutzung durch die Kooperationspartner fehlt im großen Labyrinth des Bibliotheksgebäudes bisher eine feste Anlaufstelle für die Schreibberatung. Das Netzwerk Schreiben hat sich deshalb zum Ziel gesetzt, von den vielen Einzelbuchungen der Gruppenräume abzukommen und stattdessen eigene Räume für die Schreibberatung dauerhaft zur Verfügung zu stellen.“6

An diesem Punkt der Entwicklung kommt nun das SLUB TextLab als offene Werkstatt für sämtliche Arbeiten am Text ins Spiel. Die digitale Transformation moderner Bibliotheken führt dazu, dass die physischen Bestände zugunsten elektronischer Angebote kontinuierlich zurückgehen. Damit sinkt auch der für die entsprechenden Regale benötigte Platz. Diese Entwicklung stellt Bibliotheken einerseits vor die Herausforderung, den neuen Gegebenheiten angepasste Nutzungskonzepte für ihre Standorte zu entwickeln. Andererseits schafft sie buchstäblich Freiraum für neue Ideen und neue Angebote, um auf die veränderten Bedarfe einer sich dynamisch entwickelnden Forschungs- und Universitätslandschaft einzugehen.

Vor diesem Hintergrund begannen die Fachreferenten Dr. Julia Meyer und Dr. Juan Garces, in Abstimmung mit dem SZD, im Sommer 2018 mit der Entwicklung eines neuen Standortkonzeptes für die Zweigbibliothek Erziehungswissenschaften am Standort August-Bebel-Straße, um dort einen zentralen Lern- und Schreibort als TextLab zu etablieren. ${ }^{7}$ Für den Standort sprach die räumliche Nähe zu den umliegenden Instituten der sprach- und literaturwissenschaftlichen sowie der kulturwissenschaftlichen Fakultät der TU Dresden. In Kooperation mit dem Schreibzentrum der TU Dresden (SZD) ${ }^{8}$ wurde seither im TextLab ein umfassendes Schulungs- und Beratungsangebot zum akademischen Schreiben institutionalisiert. Basierend auf der persönlichen Expertise und Qualifikation der beiden federführenden Kollegen wurde das Serviceportfolio zudem um die Facetten der Digitalen Geisteswissenschaften (Digital Humanities - DH) ${ }^{9}$ und des kreativen Schreibens erweitert. Somit wurde das SLUB TextLab zu einer offenen Werkstatt für die analoge und digitale Textarbeit, in der die Materialart Text im Mittelpunkt steht, seien es eigene Texte oder textbasierte Quellen.

6 Meyer (2018) 300.

7 Vgl. Melching und Meyer (2019) $218 \mathrm{ff}$.

8 Für eine ausführliche Darstellung der Kooperation zwischen SZD und SLUB vgl. Melching und Meyer (2019).

9 Bibliothekare verfügen durch ihre Ausbildung und berufsspezifische Erfahrung - etwa durch ihre Arbeit mit Digitalisaten, verschiedenen Standards, Schemas und Metadaten - über Kenntnisse, die auch für die Arbeit in den Digitale Humanities relevant sind. Diese Kenntnisse prädestinieren Bibliothekare geradezu zur Zusammenarbeit mit Forschern im Bereich DH. Vgl. hierzu Kijas (2017).

\section{Das TextLab nimmt Gestalt an: Neumöblierung im Jahr 2019}

Mit Beginn des Jahres 2019 begann die SLUB den Ausbau des TextLabs auch baulich voranzutreiben. Die Raumausstattung wurde aus Mitteln des Investitionsbudgets realisiert, anstehende kleinere Baumaßnahmen wurden bei der zuständigen Landesbehörde beantragt. In einem ehemaligen Garderobenraum im Erdgeschoss wurden nicht mehr benötigte Spinde abgebaut und stattdessen der Pausenraum Kaffeesatz geschaffen. Im Obergeschoss der Bibliothek wurde eine Fläche von 240 Quadratmetern neu möbliert und teilweise mit neuer Arbeitsplatztechnik ausgestattet. ${ }^{10}$ In der zweiten Jahreshälfte 2019 wurden so insgesamt 110 Arbeitsplätze geschaffen, die sich auf zwei Seminarräume, einen großen Schreibraum, einen offen gestalteten Forumsbereich mit Gruppenarbeitsplätzen und Beratungstisch, eine Leselounge und den Pausenraum verteilen. Bei der Möblierung wurde einerseits auf die Verwendung nachhaltiger und umweltgerechter Materialen Wert gelegt, andererseits auf größtmögliche Flexibilität. Klapp- und rollbare Tische und Stühle erlauben den Nutzenden die partizipative Teilnahme an der Raumgestaltung je nach ihren individuellen Anforderungen und sorgen für ergonomisches Arbeiten in vielfältigen Körperhaltungen.

Herzstück der neuen Arbeitsplatzlandschaft im TextLab ist der vollkommen neu gestaltete Forumsbereich im ehemaligen Zeitschriftensaal. Zwei Arbeitskabinen laden hier zum geschützten Arbeiten alleine oder in Kleingruppen von bis zu sechs Personen ein. Ebenfalls für Gruppenarbeiten bestens geeignet sind die beiden Stehtische mit gepolsterten Sitzbänken und Hockern, die mit Multipresenter-Displays und Anschlussmöglichkeiten für Laptop, Handy und Co. ausgestattet sind und das gemeinschaftliche Arbeiten am Text erlauben. Am Beratungstisch im Forum stehen den Nutzenden täglich Beschäftigte der SLUB und studentische Schreibtutoren des SZD für individuelle Beratung zur Verfügung. Ein Loungebereich mit Sofas und Beistelltischen ergänzt das Angebot.

Der angrenzende große Schreibraum (ehemals Zeitschriften-Lesesaal) steht all jenen offen, die konzentriert an ihren Schreibprojekten arbeiten wollen und bietet 24 Einzelarbeitsplätze. Dank der flexiblen Möblierung können die Bibliotheksbesucher den Raum gemäß ihren Be-

10 Einen Einblick mit Bildern gibt der Bericht dazu im SLUBlog (21.1.2020): https://blog.slub-dresden.de/beitrag////alles-neu-machtder-januar/. 
dürfnissen individuell gestalten - Tische und Stühle lassen sich frei nach Belieben im Raum verrücken. Für konzeptuelle Arbeiten rund um den Schreibprozess stehen ein Flipchart und eine Metaplanwand zur Verfügung, ein großes Bücherregal hält ausgewählte Ratgeberliteratur zum Schreibprozess als Präsenzbestand bereit. In einem zweiten Regal laden Meditationsbänkchen und Yogamatten und -kissen zur ausgleichenden Körperarbeit ein und unterstreichen den ganzheitlichen Ansatz des Embodiment im TextLab.

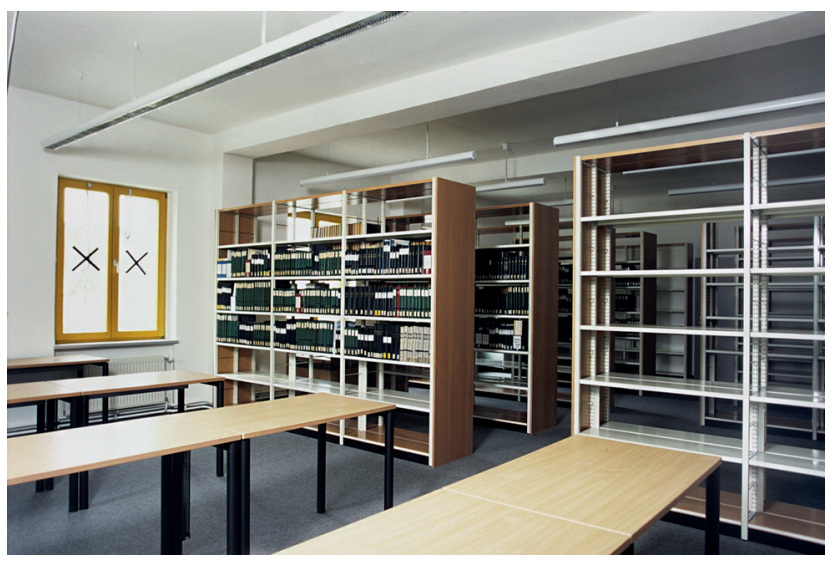

Abb. 1: Der Zeitschriftenlesesaal vor dem Umbau (Foto: SLUB Dresden / Deutsche Fotothek / Henrik Ahlers)

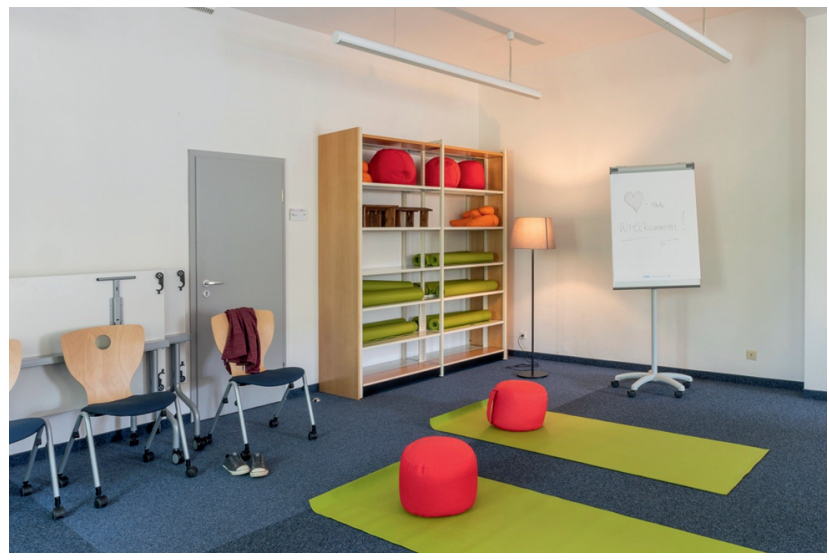

Abb. 2: Der Schreibraum heute mit Yogamatten (Foto: SLUB Dresden / Ramona Ahlers-Bergner)

Neben dem Schreibraum stehen für Gruppen von bis zu zwölf Personen zwei Seminarräume zur Verfügung, die über das Raumbuchungssystem der SLUB von allen Nutzenden selbstständig reserviert werden können. Da jede Gruppe andere Anforderungen an ihren Arbeitsplatz stellt, sind auch diese beiden Räume mit flexiblen Möbeln ausgestattet. In der warmen Jahreszeit verwandelt sich der Außenbereich des TextLab zum Grünen Schreibraum, der sowohl zum Arbeiten an der frischen Luft als auch zur Entspannung genutzt werden kann. Dafür wurden von Seiten der SLUB Gartenmöbel und Liegestühle angeschafft.

\section{3 „Wissen kommt von machen“ - das SLUB TextLab als ein Makerspace der Worte}

Vor fünf Jahren beschritt die SLUB neue Wege, ${ }^{11}$ in dem sie unter dem Leitspruch Wissen kommt von Machen ${ }^{12}$ als erste wissenschaftliche Bibliothek Deutschlands in der Zweigbibliothek DrePunct einen Makerspace als offene Werkstatt für praktisches Arbeiten und interdisziplinäre Wissensvermittlung in der Bibliothek einrichtete. Ursprünglich nur als Testballon ${ }^{13}$ gedacht, etablierte sich der Makerspace - trotz anfänglicher Skepsis - rasch als eine Fixgröße im Serviceportfolio der SLUB und feierte im Februar 2020 sein fünfjähriges Bestehen. ${ }^{14}$ Heute ist der Makerspace ein gefragter Lernort, in dem „das authentische Selber-Machen (DIY) als praktische Form des Lernens in der Bibliothek nicht zuletzt als Komplement zur Digitalität“"15 begriffen wird. Die positiven Erfahrungen bei der Durchführung von Schreibwerkstätten im Makerspace bestärkten die SLUB darin, „das Prinzip des Makerspace als Raum für prozessorientiertes Lernen und soziales Handeln über den 3D-Druck hinaus auf weitere Bereiche der Wissensvermittlung in der Bibliothek zu übertragen"16 und auf die Vermittlung von Schreibkompetenz auszuweiten. Die Analogie der zugrundeliegenden Lernkonzepte von Makerspace und Schreibwerkstatt wird in der Idee des TextLab als einem „Makerspace der Worte“ fortgeführt. Wiewohl schon oft zitiert bringt Meinhardts Beschreibung das Wesen eines Makerspace immer noch treffend auf den Punkt:

„Der eigentliche Kern des Makerspaces ist es, Dinge, Prozesse, Produkte praktisch $\mathrm{zu}$ begreifen, selbst $\mathrm{zu}$ erfahren, selbst $\mathrm{zu}$ produzieren, statt zu konsumieren - und das in einer sozialen

11 Vgl. Bonte (2016).

12 Vgl. Tiepmar et al. (2018). Für einen kritischen, wenngleich auch populärwissenschaftlichen, Artikel mit emanzipatorischen Zwischentönen zur Rolle des Machens vgl. Chachra (2015).

13 Tiepmar et al. (2018).

14 Siehe den Beitrag dazu im SLUBlog (6.2.2020): https://blog.slubdresden.de/beitrag/2020/2/6/fuenf-jahre-slub-makerspace-happy-bi rthday/.

15 Meyer (2018) 299.

16 Meyer (2018) 298. 
Umwelt, die nicht von Konkurrenz, sondern von Mitbeteiligung, Teilen und Interesse geprägt ist. Makerspaces können offensichtlich Menschen etwas (zurück)geben, das vermisst wird: Ein Verständnis dessen, warum etwas wie funktioniert, authentisches Tun, das sich nicht auf Bruchstücke beschränkt, auch Gegenständlichkeit, Sinnlichkeit, Selbstausdruck - kurz ein souveräner und unmittelbarer Zugriff auf eine immer abstraktere und vielfach als entfremdet wahrgenommene Umwelt." ${ }^{17}$

Nach dieser Definition ließe sich durchaus auch eine Schreibwerkstatt als Makerspace bezeichnen, als ein Ort in dem „[d]as Verständnis für den Schreibprozess durch das Selber-Machen und Mit-Teilen in der Gruppe praktisch erfahrbar"18 wird. Ähnlich wie im Makerspace werden auch im TextLab aus den praktischen Erfahrungen beim Erproben verschiedenster Schreibmethoden und Textsorten Erkenntnisse gewonnen und das Schreiben als eine Art Handwerk trainiert. Dabei können auch experimentelle Techniken oder kreative Schreibübungen und -spiele, die oft gar nichts mit der (akademischen) Schreibaufgabe an sich zu tun haben, dazu beitragen, den Prozess der Textgenese besser $\mathrm{zu}$ verstehen und Schreibhemmungen $\mathrm{zu}$ überwinden. Diese Erfahrungen aus erster Hand spielen eine wichtige Rolle dabei, sich der Produktion von Texten anzunähern.

Aber nicht nur bei der Textproduktion, auch bei der Beschäftigung mit textuellen Materialien in den Digitalen Geisteswissenschaften spielt das Selber-Machen eine große Rolle. Auch wenn Ramseys auf der MLA Jahrestagung 2011 in Los Angeles provokant getätigte Aussage: „Personally, I think Digital Humanities is about building things. [...] If you are not making something [...] you are not a digital humanist“19 ${ }^{\star 19}$ einer Kontroverse über das Selbstverständnis der DH-Community führte und von vielen als zu restriktiv abgelehnt ${ }^{20}$ wurde, sind Digitale Geisteswissenschaften ohne eine gewisse praktische Komponente bis heute kaum vorstellbar. Die konkrete Auseinandersetzung mit Codes, Analyse-Tools und Annotationsstandards wie der Text Encoding Initiative (TEI) hilft dabei, zugrundeliegende Prozesse besser reflektieren und verstehen zu können:

„[F]or many scholars and librarians involved in digital humanities work there is a satisfaction in the act of building or making something. Engaging with the code or tool allows you to deepen and better develop your understanding of it through hands-on application." ${ }^{21}$

17 Meinhardt (2014) 484.

18 Meyer (2018) 299.

19 Ramsey (2011b). Siehe dazu auch Ramsay (2011a).

20 Vgl. etwa Liu (2012).

21 Kijas (2017) $2 \mathrm{f}$.
Insofern lässt sich die Idee des TextLabs als einer offenen Textwerkstatt, in der Dinge selber gemacht werden, nicht nur auf das Schreiben von Texten, sondern auch auf die Beschäftigung mit Texten im Rahmen der Digitalen Geisteswissenschaften übertragen.

\section{4 „Menschen machen Bibliotheken" - Kooperation, Partizipation und Embodiment}

Getreu des in der Strategie der SLUB verankerten Grundsatzes Menschen machen Bibliotheken ${ }^{22}$ stehen auch im TextLab die Menschen im Vordergrund. Das gilt einerseits für die Beschäftigten vor Ort, die das vielfältige Angebot im TextLab ermöglichen. Dies gilt aber ebenso für die Kooperationspartner und die Nutzenden des TextLabs, die es mit ihrer Kreativität und ihrem Engagement zu dem machen, was es ist. Schließlich steht auch beim Schreibprozess im Sinne des Embodiments der Mensch in seiner Einheit von Körper, Geist und Psyche im Fokus.

Das Team des TextLab arbeitet eng mit dem bibliothekarischen Stammpersonal der Zweigbibliothek, mit weiteren Kollegen aus mehreren Abteilungen der SLUB, sowie mit externen Partnern der TU Dresden und städtischen Kultureinrichtungen zusammen. Neben den studentischen Tutoren des SZD steht dem TextLab für die Beratungstätigkeit abteilungsübergreifend eine Vielzahl an Kollegen aus den unterschiedlichsten Fachbereichen stundenweise zur Verfügung. Vor Ort wird das TextLab durch eine Koordinatorin, zwei teilgewidmete Stellen und eine freiwillige Stelle personell nachhaltig abgesichert. Seit September 2019 ist das SLUB TextLab als FSJ Einsatzstelle der Landesvereinigung Kulturelle Kinder- und Jugendbildung (LKJ) Sachsen gelistet und ermöglicht einem oder einer Jugendlichen, ein Freiwilliges Soziales Jahr Kultur im TextLab zu absolvieren. ${ }^{23}$ Zudem betreut das TextLab Abschlussprojekte hausinterner FaMIs und bietet Studierenden der Bibliothekswissenschaften die Möglichkeit eines Praxissemesters. Auf diese Art verbinden sich im TextLab traditionelle bibliothekarische Tätigkeiten mit neuen Auf-

22 Bonte und Muschalek (2019) $13 \mathrm{ff}$.

23 Für das erste Jahr konnte mit Annica Kramer eine Dresdner Abiturientin gewonnen werden, die das TextLab seit September 2019 als vollwertige Kollegin vorbildlich unterstützt und sowohl bei der Veranstaltungsorganisation als auch in der Öffentlichkeitsarbeit unerlässliche Arbeit leistet. Siehe dazu den Beitrag vom 8.4.2020: http s://textlab.hypotheses.org/2254. 
gaben zur Vermittlung von Informationskompetenz auf konstruktive Weise.

Über die SLUB hinaus stellt das TextLab den Transfer in die Dresdner Stadtgesellschaft mit starken lokalen Kooperationspartnern sicher. Im universitären Bereich sind diese Kooperationspartner vornehmlich die TU Dresden und das SZD. Aber auch abseits des universitären Settings positioniert sich das TextLab als aktiver Partner für Institutionen in der lokalen Kulturlandschaft und trägt so den außergewöhnlich breiten gesetzlichen Auftrag ${ }^{24}$ mit, den die SLUB nicht nur als Universitätsbibliothek der TU, sondern auch als Landesbibliothek Sachsens zu erfüllen hat. Auf einige Veranstaltungen in Kooperation mit der Landeshauptstadt Dresden und dem Hygiene-Museum Dresden, die den Transfer in die Stadtgesellschaft abseits der TU sicherstellen, wird in Abschnitt 5 noch näher eingegangen.

Durch die Mitgliedschaft des TextLabs in der Gesellschaft für Schreibdidaktik und Schreibforschung ist die Möglichkeit zur überregionalen Vernetzung mit anderen Schreibzentren, auch über den Raum Dresden hinaus, gegeben. Überregionale Reichweite erzielen auch die SocialMedia-Aktivitäten des TextLabs auf Twitter (@slubtextlab) und dem Blog auf textlab.hypotheses.org, die seit ihrem Start im Oktober 2019 stetig wachsende Zugriffszahlen verzeichnen (der Blog wurde im April 2020 über 1500 mal besucht). Die sozialen Medien bieten Gelegenheit zur Vernetzung im digitalen Raum und beinhalten durch die Kommentarfunktion eine starke partizipative Komponente. Auch im analogen Raum der Bibliothek laden partizipative Veranstaltungsformate wie Hackathons und Editathons, Schreibwerkstätten oder Lesekreise die Teilnehmenden dazu ein, selber kreativ tätig zu werden und ihre Ergebnisse in Form einer Ausstellung ${ }^{25} \mathrm{zu}$ präsentieren.

Vor Ort am Standort in der August-Bebel-Straße ist die Öffnung nach außen ganz konkret durch die Nutzung des Außenbereichs gegeben, der die Bibliothek für die Anrainerschaft sichtbar macht. Zudem soll der Außenbereich ab dem Frühjahr 2020 als Gemeinschaftsgarten durch den Verein Stadtgärten e.V. im Rahmen des Bürgerbeteiligungsprojektes Zukunftsstadt Dresden ${ }^{26}$ mit essbaren Pflanzen bewirtschaftet werden und damit sowohl die nachbarschaftliche Anbindung der Bibliothek stärken, als auch „einen Beitrag für die ökologisch nachhaltige Stadt

24 Vgl. Bonte (2019) 181.

25 So sollen beispielsweise Textcollagen, die von Dresdner Autorinnen im Rahmen der Schreibwerkstatt Heimat. Heute geschaffen wurden, in Form einer Wandausstellung im Pausenraum gezeigt werden. 26 Siehe dazu Landeshauptstadt Dresden, Projekt Zukunftsstadt: htt ps://www.zukunftsstadt-dresden.de/was-ist-die-zukunftsstadt/. der Zukunft“ ${ }^{\star 27}$ leisten. Zur Realisierung des Projektes müssen jedoch vorab sowohl die baulichen Rahmenbedingungen geschaffen werden, wozu die SLUB auf die Kooperation der zuständigen Behörden angewiesen ist, als auch eine Reihe von Erfolgsfaktoren erzielt werden. Diese „sind selten themenspezifisch, sondern gelten meist für Bibliotheksinnovationen im weiteren Sinne" ${ }^{\text {28 }}$ und schließen u. a. mit ein, nachhaltig zu planen, Grenzen klar zu definieren und Akzeptanz für das Projekt in und außerhalb der Bibliothek zu gewinnen. ${ }^{29}$

Schon jetzt kann der Außenbereich des TextLab in der warmen Jahreszeit mit seinen Klapptischen und -stühlen als grüner Schreibraum genutzt werden. Liegestühle laden zur Entspannung ein, auch die Yogamatten und Kissen aus dem Schreibraum können mit ins Freie genommen und dort zur Meditation oder Entspannung genutzt werden. Hintergrund dieser Angebote ist der ganzheitliche Ansatz des Embodiment, ${ }^{30}$ der das Zusammenspiel von Geist, Körper und Psyche in den Mittelpunkt rückt und auch in der Schreibforschung immer mehr Berücksichtigung findet. Jüngere Studien untersuchen Embodied Writing sowohl im Kontext des Kreativen Schreibens ${ }^{31}$ als auch in der akademischen Schreibberatung ${ }^{32}$ und belegen, dass Schreiben niemals eine reine Geistesarbeit ist, sondern immer auch als ein körperlicher Prozess begriffen werden muss. Der Schriftsteller Kurt Drawert bringt diese Tatsache sehr bildhaft zum Ausdruck, wenn er schreibt: „Der Text ist ein symbolisches und ein physisches Ereignis. Ihn zu schreiben strengt an, körperlicher, als man es gemeinhin annehmen mag [...] eine Art Steinmetzarbeit im Alphabet.“33

Dementsprechend stehen den Besuchern des TextLabs ein eigener Pausenraum und eine Leselounge zur Verfügung, die Möglichkeiten zur Rekreation zwischen intensiven Lern- und Schreibphasen bieten und das TextLab als Dritten Ort im Tagesablauf der Nutzenden verankern. Yogamatten- und Kissen im Schreibraum laden zur ausgleichenden Körperarbeit ein, die für nachhaltiges Lernen unabdingbar ist. Als Akteur im Netzwerk achtsamer Hochschulen in der digitalen Gesellschaft ${ }^{34}$ bietet das TextLab zudem in Kooperation mit dem Universitätsportzentrum

27 Landeshauptstadt Dresden, Projekt Essbares öffentliches Stadtgrün - bürgerschaftlich gepflegt: https://www.zukunftsstadt-dresden. de/projekte/f1/.

28 Bonte (2016) 91.

29 Vgl. Bonte (2016) $91 \mathrm{ff}$.

$30 \mathrm{Vgl}$. Storch et al. (2010).

31 Vgl. Sultan (2018).

32 Vgl. Clayson (2018).

33 Drawert (2012) 64.

34 Näheres zu dieser Initiative bietet der Webauftritt unter https://ac htsamehochschulen.de/. 
und dem Gesundheitsmanagement der TU Dresden Yoga und Entspannungskurse in den beiden Gruppenräumen an, die regen Zulauf erfahren.

\section{Mehr als nur Schreiben - Das Beratungs- und Veranstaltungsangebot im SLUB Textlab}

Das Verfassen (wissenschaftlicher) Texte ist eine wichtige Schlüsselkompetenz im Studium und darüber hinaus auch in der Berufswelt für Akademiker. Trotzdem werden Herangehensweisen an das Schreiben nicht in jedem Studiengang hinreichend thematisiert und oft nicht systematisch vermittelt. ${ }^{35}$ Ein gemeinsames Ziel von SLUB und Schreibzentrum der TU (SZD) ist es daher, „Schreibfähigkeiten zu stärken und das Bewusstsein dafür zu schaffen, dass das akademische Schreiben erlernbar ist und trainiert werden muss“. ${ }^{36}$ Dazu gibt es im SLUB TextLab ein vielfältiges Schulungs- und Beratungsangebot, das sich primär an Studierende richtet (siehe Abschnitt 5.1). Das TextLab bietet allerdings noch um einiges mehr. Als Research-Support-Dienstleister und Projektpartner für die digitalen Geisteswissenschaften stellt das TextLab Expertise und Räumlichkeiten für umliegende Institute der TU Dresden bereit (Abschnitt 5.2). Aber auch über das universitäre Umfeld hinaus positioniert sich das TextLab als aktiver Partner für Institutionen in der Kulturlandschaft Dresdens (siehe Abschnitt 5.3). Dieses vielseitige Angebot stößt auf positive Resonanz bei den Nutzenden: Monatlich nehmen bis zu 700 Personen an Veranstaltungen im SLUB TextLab teil.

\subsection{Schreiben im wissenschaftlichen Kontext}

Ein Gutteil der Beratungs- und Schulungsangebote im TextLab richtet sich an Studierende. Ergänzend zu einem vielseitigen Workshop- und Veranstaltungsangebot findet in Kooperation mit dem SZD täglich Schreibberatung in der offenen Textwerkstatt im Forum statt. Dabei ergänzen sich die Angebote der SLUB und des SZD: Während die SLUB vorwiegend facheinschlägige Beratung durch Fachreferenten und sonstige Experten bietet, ist die stu-

35 Vgl. Melching und Meyer (2019) 211.

36 Melching und Meyer (2019) 211. dentische peer-to-peer-Beratung des Schreibzentrums fachunspezifisch. ${ }^{37}$ In Summe bietet das TextLab damit umfassenden Service zum akademischen Schreiben. Schreibsprechstunden können von den Nutzern entweder spontan bei Bedarf wahrgenommen oder online gebucht werden, damit sich die Berater auch komplexeren Fragestellungen widmen können, die mitunter etwas Vorbereitung erfordern.

Oft steht in der Schreibberatung gar nicht der Text selbst, das Produkt, im Vordergrund, sondern der Weg dahin, der Prozess. ${ }^{38}$ Das beginnt bei der Wahl und Eingrenzung eines geeigneten Themas und endet bei der stilistischen und sprachlichen Überarbeitung des Rohtextes. Neben der Beratung zu den verschiedenen Phasen im Schreibprozess bilden aber auch äußere Einflussfaktoren Beratungsschwerpunkte: Wie wirke ich Ablenkungen beim Schreiben entgegen? Was sind meine Stärken und Schwächen beim Schreiben? Wie kann ich Schreibhemmungen überwinden? All das sind Fragen, derer sich die studentischen Schreibtutoren in den 45 bis 60 Minuten eines Beratungstermins annehmen. Für tiefergehende Anliegen bezüglich Recherchestrategien, Suchwortgenerierung in Datenbanken oder der Verwendung von Literaturverwaltungsprogrammen verweisen die studentischen Schreibtutoren an die Experten der SLUB im TextLab, die fachspezifisch beraten und auf weitere Angebote der SLUB $\mathrm{zu}$ Themen wie Literaturverwaltung oder Zitieren verweisen. Durch die bestehende Kooperation zwischen SLUB und Schreibzentrum, die seit Juli 2019 auch schriftlich in einer Kooperationsvereinbarung fixiert ist, kann das TextLab zu nahezu allen Fragen rund um das Verfassen wissenschaftlicher Arbeiten Beratung unter einem Dach bieten.

Ausschlaggebend für die Qualitätssicherung und damit letztlich auch für den Erfolg der Beratung ist der kontinuierliche, kollegiale Austausch zwischen den Kooperationspartnern. Denn nur wenn ,die in der Schreibberatung tätigen Mitarbeiterinnen und Mitarbeiter Einblick in die Beratungspraxis des jeweiligen Kooperationspartners erhalten, sinnvoll aufeinander verweisen und Fragen und Probleme gemeinsam erörtern können “, ${ }^{39}$ kann auch die Qualität der Beratungen und die sinnvolle Komplementarität des Angebots garantiert und transparent an die Nutzenden kommuniziert werden. Ein erster Schritt in Richtung eines systematischen Hospitationsprogrammes wurde im Dezember 2019 mit einer gemein-

37 Zur Schreibberatung im TextLab siehe auch Melching und Meyer (2019) $213 \mathrm{ff}$.

38 Melching und Meyer (2019) 214.

39 Melching und Meyer (2019) 222. 
samen Team-Fortbildung unternommen. Dabei trafen sich die knapp vierzig Beteiligten zu einem halbtägigen Workshop, der dem persönlichen Kennenlernen, dem fachlichen Austausch sowie dem gegenseitigen Präsentieren der unterschiedlichen Beratungsangebote diente. Dieses Format soll im Team gemeinsam weiterentwickelt und dann regelmäßig zweimal jährlich durchgeführt werden, jeweils als Vorbereitung für die beratungsintensive Zeit zum Ende des Semesters und Start der Prüfungsphase.

Zur internen Weiterentwicklung als Team kommt der gemeinsame Auftritt des TextLab nach außen hinzu. Einen wichtigen Schritt in dieser Beziehung stellte die sehr publikumswirksame Lange Nacht des Schreibens dar, die seit mehreren Jahren am ersten Donnerstag im März an vielen Hochschulen und Schreibzentren im deutschsprachigen Raum zeitgleich begangen wird..$^{40}$ Sie fand 2020 erstmalig im TextLab statt und bot eine schöne Gelegenheit, die neu ausgestatteten Räumlichkeiten einer breiteren Öffentlichkeit zu präsentieren. Unter dem Motto Write through the night trafen sich ca. 200 Personen, um gemeinsam an Texten aller Art zu schreiben. Die Veranstaltung wurde von einem vielfältigen Rahmenprogramm begleitet. So konnten sich die Teilnehmenden der sogenannten PreNight bereits ab 16 Uhr in insgesamt vier ausgebuchten Workshops à 90 Minuten mit Themen wie Lost in Literature - Wie finde ich den roten Faden? auseinandersetzen. Ab 18 Uhr standen Mitarbeiter der SLUB für individuelle Beratung zu den Themen wie Literaturrecherche in Datenbanken oder Schreiben mit MS Word zur Verfügung und ergänzten auf diese Art die Peer-Beratung des Schreibzentrums. Der Schreibraum, die beiden Seminarräume und der Eltern-Kind-Raum der Bibliothek wurden für Schreibgruppen genutzt, in denen verschiedenen Methoden zum wissenschaftlichen Schreiben oder zum Zeitmanagement ausprobiert werden konnten. Um den ökologischen Aspekt der Veranstaltung zu unterstreichen, war die TU Umweltinitiative mit einem eigenen Stand vertreten und sorgte mit dem Smoothie-Fahrrad, auf dem man sich durch einen mit Muskelkraft angetriebenen Mixer Fruchtshakes erstrampeln konnte, für den nötigen körperlichen Ausgleich zwischen den intensiven Arbeitsphasen. Ebenso großer Beliebtheit erfreute sich das zu jeder vollen Stunde für 15 Minuten im Forum angebotene Schreibtisch-Yoga. Der partizipative Charakter der Veranstaltung wurde durch eine Schreibimpulswand, die zum Austausch von Schreibtipps einlud, den Schreibtypen-Test und den Schreibprozess-Walk, bei dem die Teilnehmenden die ver-

40 Zum Format der Langen Nacht des Schreibens siehe Melching und Meyer (2019) 216 sowie Girgensohn et al. (2019). schiedenen Phasen des Schreibprozesses auch räumlich nachvollziehen konnten, noch zusätzlich unterstrichen.

\subsection{Digital Humanities (DH)}

Zu den zentralen Fragestellungen im Rahmen der Beschäftigung mit dem Rohstoff Text im TextLab gehören unter anderem die Reflexion und das Wissen darüber, wie Texte im Schreibprozess entstehen, wie sie stilistisch überarbeitet werden können und wie Schreiben dabei helfen kann, unser Denken und Lernen zu unterstützen. Ebenso im Fokus stehen aber auch Möglichkeiten, Quellentexte digital zu erfassen und mit Informationen anzureichern. Als Konsequenz daraus ergibt sich die Frage nach geeigneten Methoden zur computergestützten Analyse dieser Texte und nach der visuellen Präsentation der dabei gewonnenen Forschungsergebnisse. In der TEI-Werkstatt im TextLab lernen Teilnehmende zum Beispiel, Textquellen mit dem XML-Oxygen-Editor nach dem Annotationsstandard der Text Encoding Initiative auszuzeichnen, um sie anschließend in der Programmiersprache Python zu analysieren. Ebenso finden korpuslinguistische Workshops und Seminare in Zusammenarbeit mit Lehrenden der TU Dresden verschiedener Institute im Bereich Geistes- und Sozialwissenschaften statt.

Das SLUB TextLab versteht sich hierbei als ResearchSupport-Dienstleister für die Digitalen Geisteswissenschaften und bietet ein reiches Programm an Beratungen, Workshops und Seminaren für Studierende und Lehrende gleichermaßen an, das von der Erstellung von Digitalisaten über OCR-Software Entwicklung bis hin zum digitalen Edieren und Analysieren von Texten ein breites Spektrum abdeckt. Dabei organisiert das TextLab auch relativ kurzfristig und bedarfsgerecht Workshops mit internen oder externen Experten. So fand im Wintersemester 2019/20 ein Workshop zum Thema Statistik mit Python für Geisteswissenschaftler statt. Für das Sommersemester 2020 ist ein Seminar zum selbstständigen erstellen hochwertiger OCRErkennung sowie zur Korpuslinguistik mit der Python-Bibliothek NLTK geplant.

Neben der Beratung zu technischen Fragen besteht ein wichtiger Aspekt dieses forschungsunterstützenden Service auch in der Unterstützung bei der Planung von Forschungsprojekten und der Drittmittel-Akquise für deren Umsetzung. Hier tritt das TextLab mitunter auch als Projektpartner auf, wie etwa in den Projekten Marcion Bible ${ }^{41}$

41 Nähere Informationen zum Projekt unter https://marcionbible.tudresden.de/. 
und DigitaLIS - Digitale Literatur- und Sprachwissenschaf ten. Dem Projekt DigitaLIS kommt hier besondere Relevanz zu, dient es doch der Vorbereitung eines geplanten MA-Studiengangs Digital Humanities an der TU Dresden, der im Wintersemester 2020/21 starten soll und die Angebote des TextLab auch curricular an der Universität verankern wird. Das Projekt wird durch das Sächsische Staatsministerium für Wissenschaft, Kultur und Tourismus (SMWK) im Rahmen des Digital Fellowship-Programms für die Weiterentwicklung der digitalisierten Hochschulbildung gefördert. Es startete mit Oktober 2019 und hat eine Laufzeit von zwei Jahren.

Ein erster Testlauf im Wintersemester 2019/20 umfasste die Vorlesung Digital gestützte Analyse in der Historischen Linguistik von Alexander Lasch (Institut für Germanistik), das Seminar Digitales Edieren in den Literaturwissenschaften von Juliane Rehnolt (Institut für Slavistik) und Juan Garcés (SLUB TextLab), sowie die Digitalisierungswerkstatt Digital Humanities. Einführung in die Erschließung und Analyse von Texten und Bildern von Juan Garcés (SLUB TextLab). Diese Lehrveranstaltungen wurden im Zuge des Projektes einerseits für den neuen Masterstudiengang pilotiert und von studentischen Hilfskräften dokumentiert, sowie andererseits hochschuldidaktisch durch Anja Swidsinski vom Projekt LiT - Lehrpraxis im Transfer begleitet und evaluiert. Gemeinsam stellen sich somit die TU Dresden, die professionelle Lehrberatung und das SLUB TextLab der Herausforderung, ein stimmiges Konzept für zukünftige Lehre zu entwickeln. ${ }^{42}$ Übergeordnetes Ziel des Projektes ist es, nachhaltig eine curriculare Einheit für den Masterstudiengang DH aufzubauen und dabei ,verschiedene Lehrveranstaltungen miteinander zu verzahnen und digitale Methoden nachhaltig in der Lehre zu verankern“. ${ }^{43}$ Das SLUB TextLab stellt dabei sowohl den Veranstaltungsort als auch mit Dr. Juan Garces einen der Dozenten, der in Eigenverantwortung und im Team mit Lehrenden der TU die Kurse abhält. In diesem Sinn wird das TextLab dem neuen Studiengang auch ganz konkret ein Zuhause bieten.

\subsection{Außeruniversitärer Transfer: das SLUB Textlab als Akteur in der Dresdner Kulturlandschaft}

Bei aller Anbindung an die TU Dresden und ihrer Bedarfe erfüllt die SLUB in ihrer Rolle als Staats- und Landesbiblio-

42 Vgl. Swidsinski und Rehnolt (2020).

43 Swidsinski und Rehnolt (2020) o. S. thek Sachsens aber auch einen gesetzlichen Auftrag über diese Klientel hinaus. Mit seinem breit gefächerten Veranstaltungsangebot möchte auch das Textlab eine möglichst große Nutzerschicht ansprechen. In Kooperation mit der Landeshauptstadt Dresden, die sich für den Titel Kulturhauptstadt Europas 2025 bewarb, schrieb die SLUB im März 2019 einen Wettbewerb für Nachwuchsautoren aus. ${ }^{44}$ Aus über 60 Bewerbungen aus ganz Deutschland wurden im Juni 2019 zwei Gruppen von Autoren ausgewählt, die unter der Leitung von Schriftsteller Kurt Drawert und Dr. Julia Meyer, Fachreferentin für Germanistik, zwischen September 2019 und Mai 2020 in einer Reihe von Schreibwerkstätten an ihren Texten ${ }^{45}$ arbeiteten. Begleitend zu den Treffen der Schreibwerkstätten, die teils im TextLab und teils in anderen Räumlichkeiten der SLUB stattfanden, wurden die Autoren auf dem Blog des TextLab vorgestellt und erhalten dort eine Plattform, um kurze literarische Texte zu präsentieren. Durch die Dresdner Autorin Cornelia Eichner, Teilnehmerin an der Schreibwerkstatt, ergibt sich damit auch die Verbindung zum Literaturnetz Dresden, das auf Initiative von Cornelia Eichner 2018 gegründet wurde.

Ebenfalls einen starken regionalen Bezug hat die bürgerwissenschaftlichen Veranstaltungsreihe Vergessene Dresdner Schriftstellerinnen. ${ }^{46}$ Die Autorin und Musikerin Uta Hauthal forschte in den Archivbeständen der SLUB zu fünf Dresdner Schriftstellerinnen des 20. Jahrhunderts. Um ihre Forschungsergebnisse der Öffentlichkeit zugänglich zu machen und die Autorinnen und ihre Werke vor dem Vergessen zu bewahren, konzipierte Hauthal in Kooperation mit dem SLUB TextLab und dem Dresdner Geschichtsverein eine Veranstaltungsreihe, die zwischen Januar und Mai 2020 monatlich eine der Schriftstellerinnen im Vortragssaal der SLUB präsentieren sollte. Die vorgestellten Bücher sollten in begleitenden Lesekreisen diskutiert werden. Aufgrund des Ausbruchs der Corona-Pandemie im März 2020 musste die Reihe nach zwei Abenden unterbrochen werden. Um dem interessierten Publikum auch während der Kontaktsperre einen Abend mit Uta

44 Siehe dazu den Beitrag im SLUBlog (10.7.2019): https://blog.slubdresden.de/beitrag////schreibwerkstatt-heimat-neu-denken-teilneh merinnen-stehen-fest/.

45 Einer der Texte, der bereits im September 2019 in der Schreibwerkstatt diskutiert wurde, ist Amanda Lasker-Berlins Debütroman Elijas Lieds, der im Frühjahr 2020 bei der Frankfurter Verlagsanstalt erschien und in mehreren Rezensionen als überzeugender, politischer Roman gewürdigt wurde. Elijas Lied ist für den Debütpreis der lit. COLOGNE 2020 nominiert.

46 Siehe dazu den Beitrag im SLUBlog (24.1.2020): https://blog.slubdresden.de/beitrag////vergessene-dresdner-schriftstellerinnen-ein-a bend-ueber-johanna-lankau-in-der-slub/. 
Hauthal bieten zu können, produzierte die SLUB im Mai einen knapp halbstündigen Videogruß aus dem Klemperer Saal. ${ }^{47}$ Die begleitenden Lesekreise haben als virtuelle Literaturzirkel auf dem Blog des TextLabs eine digitale Heimat gefunden und beinhalten mittlerweile sogar kreative Schreibaufgaben für die Teilnehmer. Mit Anfang Juni 2020 sollen nun auch die geplanten literarischen Spaziergänge starten, bei denen Uta Hauthal an Originalschauplätzen aus den Werken der Schriftstellerinnen liest und die vom Kulturamt der Landeshauptstadt Dresden gefördert werden.

Neben der Landeshauptstadt Dresden hat das TextLab mit der Kustodie der TU Dresden und dem Deutschen Hygiene-Museum noch zwei weitere gewichtige Akteure in der lokalen Kulturszene zum Kooperationspartner. Hier verdienen insbesondere die kreativen Schreibwerkstätten eine Erwähnung, die Dr. Julia Meyer seit einigen Jahren begleitend $\mathrm{zu}$ den halbjährlich wechselnden Ausstellungen des Hygiene-Museums organisiert. Die Schreibwerkstätten werden als Weiterbildungsangebot der sächsischen Bildungsagentur für Lehrer angeboten, die ihre Erfahrungen dann im kreativen Schreibunterricht weitergeben können. Ausgehend vom Thema der jeweils aktuellen Ausstellung treffen sich die Teilnehmenden abwechselnd im TextLab und im Hygiene-Museum. Das Nebeneinander von Kunst und Literatur beflügelt - im kreativen Dialog mit den Exponaten und den Besuchern der Ausstellung entstehen so mitunter experimentelle Texte, etwa aus der narrativen Perspektive eines Tieres im Rahmen der Ausstellung Tierisch beste Freunde über Haustiere. Die aktuelle Schreibwerkstatt zum Thema $\mathrm{Na}$ ture Writing, die begleitend zur Sonderausstellung Von Pflanzen und Menschen für April $2020^{48}$ geplant war, musste aufgrund der aktuellen Situation leider abgesagt werden.

\section{Fazit und Ausblick}

Mit seiner Kombination von Schreibberatung, Digital Humanities und Embodiment bietet das SLUB TextLab ein innovatives und vielseitiges Angebot zum Schreiben und zu den Digitalen Geisteswissenschaften, das von den Nutzenden sehr gut angenommen wird und sich bereits nach den ersten Monaten in einer deutlich gesteigerten Auslastung der Bibliothek niederschlägt. Kooperationen mit uni-

47 Das Video (14.5.2020) ist abrufbar unter https://blog.slub-dres den.de/beitrag/2020/05/14/gruss-aus-dem-klemperer-saal-vergesse ne-dresdner-schriftstellerinnen-mit-uta-hauthal/.

48 Vgl. dazu SLUB Dresden (2020). versitären und starken außeruniversitären Partnern wie der Landeshauptstadt Dresden und dem Deutschen Hygiene-Museum Dresden sichern nachhaltig das Angebot im TextLab und den Transfer in die Stadtgesellschaft. Um das Alleinstellungsmerkmal des ganzheitlichen Ansatzes im Sinne des Embodiments in Zukunft noch stärker zu betonen, soll im Außenbereich des TextLab ein Gemeinschaftsgarten realisiert werden. Ebenso soll die räumliche Nähe zum Großen Garten, einem weitläufigen Park im Zentrum Dresdens, in Outdoor-Formaten wie einer Spazierschreibsprechstunde und einer angeleiteten Auszeit im Grünen zwischen intensiven Schreibphasen verstärkt genutzt werden. Im Bereich der Digitalen Geisteswissenschaften ist mit der geplanten Einführung eines Masterstudiengangs DH an der TU Dresden ab dem Wintersemester 2021/22 die curriculare Verankerung der Angebote des TextLabs als nächster Schritt bereits konkret angedacht. Die Idee der Partizipation ist im Konzept des TextLabs fest verankert und entspricht dem in der Strategie SLUB 2025 definierten Ziel Wissen teilen - Menschen verbinden. ${ }^{49}$ Durch wechselnde Ausstellungen im Pausenraum, die etwa die Ergebnisse der kreativen Schreibwerkstätten präsentieren könnten, soll dieser partizipative Charakter noch stärker betont werden.

\section{Literaturverzeichnis}

Bonte, Achim (2016): Vorstoß in neue Wissensräume. Makerspaces im Leistungsangebot wissenschaftlicher Bibliotheken. In: Strategien für die Bibliothek als Ort, hg. v. Konrad Umlauf, Klaus Ulrich Werner und Andrea Kaufmann, 85-94. DOI: 10.1515/ 9783110481037-006.

Bonte, Achim (2019): Ein offener Wissensmarktplatz mit gesellschaftlicher Relevanz. Perspektiven der SLUB im digitalen Zeitalter. In: BIBLIOTHEK - Forschung und Praxis, 43 (1), 180-84. DOI: https://doi.org/10.1515/bfp-2019-2022.

Bonte, Achim; Muschalek, Antonie (Hrsg.) (2019): Wissen teilen Menschen verbinden. SLUB 2025. Strategie der sächsischen Landesbibliothek - Staats- und Universitätsbibliothek Dresden. Dresden: Sächsische Landesbibliothek, Staats- und Universitätsbibliothek. Verfügbar unter https://nbn-resolving.org/urn: nbn:de:bsz:14-qucosa2-357501.

Chachra, Debbie (2015): Why I am not a Maker. In: The Atlantic, 23. January 2015. Verfügbar unter https://www.theatlantic.com/ technology/archive/2015/01/why-i-am-not-a-maker/384767/.

Clayson, Ashley (2018): Distributed writing as a lens for examining writing as embodied practice. In: Technical Communication Quarterly, (3), 217-26.

Drawert, Kurt (2012): Schreiben. Vom Leben der Texte. München: C.H. Beck.

49 Vgl. Bonte \& Muschalek (2019). 
Girgensohn, Katrin; Hertz-Eichenrode, Hans-Jürgen; Voigt, Anja (2019): Die Lange Nacht der aufgeschobenen Hausarbeiten Wissenschaftliches Schreiben als Gemeinschaftserlebnis zwischen Pyjama-Party und Klosterstille. In: Praxishandbuch Schreiben in der Hochschulbibliothek, hg. v. Wilfried Sühl-Strohmenger und Ladina Tschander. Berlin; Boston: De Gruyter, 121-29.

Kijas, Anna E. (2017): An Introduction to Getting Started in the Digital Humanities for Library Professionals. In: Music Reference Services Quarterly, 20 (1), 1-7, DOI: 10.1080/10588167.2017. 1274595.

Liu, Alan (2012): Where is cultural criticism in the Digital Humanities? In: Debates in the Digital Humanities, hg. v. Matthew K. Gold, Minneapolis: University of Minnesota Press, 490-510.

Meinhardt, Heike (2014): Das Zeitalter des kreativen Endnutzers. Die Lernlab-, Creatorspace und Makerspace-Bewegung und die Bibliotheken. In: BuB - Forum Bibliothek und Information, 66 (6), 479-85.

Melching, Nina; Meyer, Julia (2019): Netzwerk Schreiben. Kooperation zwischen dem Schreibzentrum der TU Dresden und der SLUB Dresden. In: Praxishandbuch Schreiben in der Hochschulbibliothek, hg. v. Wilfried Sühl-Strohmenger und Ladina Tschander. Berlin, Boston: De Gruyter, 210-22. DOI: https://doi.org/ 10.1515/9783110594140-020.

Meyer, Julia (2018): Kafka im Makerspace. Kooperation in der akademischen Schreibberatung. In: Kooperative Informationsinfrastrukturen als Chance und Herausforderung, hg. v. Achim Bonte und Juliane Rehnolt. Berlin, Boston: De Gruyter, 289-301. DOI: https://doi.org/10.1515/9783110587524-032.

Mittelbach, Jens (2013): Eine Wissensbar für die SLUB: Informationsvermittlung als echte Dienstleistung. In: BIS - Das Magazin der Bibliotheken in Sachsen, 6 (3), 180-83.

Ramsay, Stephen (2011a): On building. Blogbeitrag vom 11.1.2011 auf http://lenz.unl.edu/papers/2011/01/11/on-building.html. Abgedruckt in: Defining Digital Humanities. A Reader (2013), hg. v. Melissa Terras, Julianne Nyhan und Edward Vanhoutte, Kap. 14. Farnham: Ashgate.

Ramsey, Stephen (2011b): Who's in and who's out?. Vortrag am 7.1.2011 im Panel „The History and Future of the Digital Humanities" auf der Jahrestagung der Modern Languages Association (MLA): Defining Digital Humanities. A Reader (2013), hg. von Melissa Terras, Julianne Nyhan und Edward Vanhoutte, Kap. 13. Farnham: Ashgate.
Santner, Anita (2020): Vor den Vorhang: „Wer bloggt hier eigentlich?“ - Annica Kramer im Porträt. Verfügbar unter https://textlab.hypotheses.org/2254.

SLUB Dresden (2020): Nature Writing. Verfügbar unter https://www. slub-dresden.de/service/slub-textlab/nature-writing/.

Storch, Maja; Cantieni, Benita; Hüther, Gerald; Tschacher, Wolfgang u. a. (Hrsg.) (2010): Embodiment. Die Wechselwirkung von Körper und Psyche verstehen und nutzen. Bern: Hans Huber.

Sultan, Nevine (2018): Embodied Self-Care: Enhancing Awareness and Acceptance Through Mindfulness-Oriented Expressive Writing Self-Disclosure. In: Journal of Creativity in Mental Health, (1), 76-91.

Swidsinski, Anja; Rehnolt, Juliane (2020): Vorhang auf für „DigitaLiS“: Ein kleiner Einblick in die Studiengangsentwicklung Master Digital Humanities an der TU Dresden. Verfügbar unter https:// textlab.hypotheses.org/2342.

Tiepmar, Jonas; Mittelbach, Jens; Kaiser, Melanie; Dobeleit, Daniela; Schwanse, Paul; Fröhner, Uta; Jähne, Maik (2018): Wissen kommt von Machen. Zukunftsgestaltung in wissenschaftlichen Bibliotheken im Zeitalter der Digitalisierung. In: BIBLIOTHEKForschung und Praxis, 42 (1), 69-82, DOI: https://doi.org/ 10.1515/bfp-2018-0009.

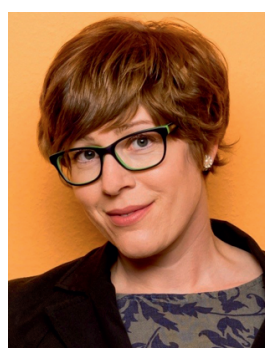

\section{Anita Santner}

Sächsische Landesbibliothek - Staats- und Universitätsbibliothek Dresden (SLUB)

D-01054 Dresden

anita.santner@slub-dresden.de 